\title{
Geotechnical Engineering: Referees 2010
}

The following is a list of referees who have reviewed papers for Geotechnical Engineering between 1 January 2010 and 31 December 2010. The Institution of Civil Engineers is very grateful for their assistance.

We are continually looking for suitable reviewers for papers submitted to Geotechnical Engineering. Papers published in the Proceedings of the ICE must be submitted to at least two independent referees to judge accuracy, style, impact, importance and interest.

\begin{tabular}{|c|c|c|c|c|}
\hline D. Allenby & A. Chodorowski & D. Greenwood & N. Matasovic & N. Shirlaw \\
\hline A. Al-Tabbaa & D. Choudhury & J. Greenwood & M. Maugeri & D. Shohet \\
\hline C. Anagnostopoulos & F. C. Chow & S. Hake & B. McCabe & G. Shore \\
\hline M. Arnold & J. Chu & J. Hall & P. McCombie & S. K. Shukla \\
\hline A. Arulrajah & B. Clarke & M. Hall & A. McNamara & I. Sims \\
\hline J. H. Atkinson & J. Colmenares & C. Hartley & P. Meijers & N. Sitar \\
\hline C. Augarde & N. Consoli & K. Hawkins & R. Meriggi & N. Sivakugan \\
\hline B. Avar & J. Cook & C. Howley & C. Merrifield & P. V. Sivapullaiah \\
\hline F. Badelow & S. Corbet & Y. S. Hsu & A. Merritt & H. Skinner \\
\hline C. Barker & F. Cotecchia & P. Hughes & G. Milligan & J. Smethurst \\
\hline F. Basile & A. Cresswell & E. Ibraim & T. Miranda & D. Smith \\
\hline D. Beadman & J. Cripps & R. Jardine & E. Mohamedelhassan & P. Smith \\
\hline K. S. Beena & M. Czerewko & A. Javadi & D. Muir Wood & K. Soga \\
\hline L. Begaj Qerimi & A. Dawson & S. Jefferis & S. A. Naeini & M. Srbulov \\
\hline S. Bhattacharya & M. Devriendt & C. Jenner & T. Newson & J. Steenfelt \\
\hline A. Binns & L. Di Matteo & R. Jimenez & K. Nicholls & D. Stewart \\
\hline A. Bond & P. Doherty & C. Jommi & A. Nooy van der Kolff & K. Stone \\
\hline P. Bourne-Webb & Y. Doulala-Rigby & B. Jones & P. Nowak & A. M. Tang \\
\hline N. Boylan & M. Dyer & C. J. F. P. Jones & S. Olivella & R. Thompson \\
\hline A. Brennan & A. El Nahas & M. Kahyaoglu & C. O'Loughlin & D. Toll \\
\hline K. Briggs & G. Elia & M. Khan & M. O’Neill & D. Tonks \\
\hline E. Bromhead & E. Ellis & J. Knappett & J. Oti & I. Tromans \\
\hline S. Brown & S. Endley & S. Kontoe & J. Palmer & S. Utili \\
\hline D. Bruce & M. England & N. Kovacevic & E. Passaris & A. Valsangkar \\
\hline G. Bunce & R. J. Fannin & J. Kupec & A. Pellew & V. van Beek \\
\hline H. J. Burd & E. Farrell & P. V. Lade & J.-M. Pereira & C. Viggiani \\
\hline S. Buttling & C. Fenton & C. Lambert & A. Phear & S. Walthall \\
\hline B. Byrne & A. Flora & J. Lee & P. Pinto & K. Watts \\
\hline F. Camargo & R. Flynn & E. C. Leong & W. Powrie & S. Wersching \\
\hline T. Carrington & S. Foti & V. Li & M. M. Rahman & C. White \\
\hline H.-Y. Chai & A. Gaba & D. Lo Presti & D. Richards & I. Whyte \\
\hline A. Chan & M. Gambin & M. Long & E. Rojas & M. Winter \\
\hline T. Chapman & J. Gannon & F. Loveridge & P. Rutty & J. Yiu \\
\hline A. Charles & K. Gavin & Z. Lubkowski & R. Sandham & L. Zdravkovic \\
\hline J. Charrach & V. Germano & A. Lutenegger & V. Saouma & A. Zervos \\
\hline R. Chen & S. Goren & M. Madhav & F. Schroeder & C. Zhao \\
\hline K. F. Cheung & S. Gourvenec & G. Manfredi & H. Schweiger & \\
\hline E. L. Chin & J. Graham & F. Marinho & J. Sharma & \\
\hline
\end{tabular}

If you are interested in reviewing articles on any topic related to geotechnical engineering, please submit your name, qualifications or $\mathrm{CV}$, and areas of expertise. We are in need of individuals who will agree to review papers in a timely fashion (within 3 to 4 weeks of receipt) and provide confidential feedback to the Editorial Advisory Panel concerning the quality of the paper and any suggested revisions that would be appropriate.

If you are such a person, please contact Sohini Banerjee (tel.: +44 (0)207 665 2476; e-mail: sohini.banerjee@ice.org.uk) for more information on the referee process. 